\title{
NGC 2419-ANOTHER REMNANT OF ACCRETION BY THE MILKY WAY*
}

\author{
Judith G. Cohen ${ }^{1}$, Evan N. Kirby ${ }^{1,4}$, Joshua D. Simon ${ }^{2}$, and Marla Geha ${ }^{3}$ \\ ${ }^{1}$ Palomar Observatory, Mail Stop 249-17, California Institute of Technology, Pasadena, CA 91125, USA; jlc(enk)@ astro.caltech.edu \\ ${ }^{2}$ Carnegie Observatories, 813 Santa Barbara Street, Pasadena, CA 91101, USA; jsimon@obs.carnegiescience.edu \\ ${ }^{3}$ Astronomy Department, Yale University, New Haven, CT 06520, USA; marla.geha@yale.edu \\ Received 2010 June 4; accepted 2010 September 29; published 2010 November 17
}

\begin{abstract}
We isolate a sample of 43 upper red giant branch stars in the extreme outer halo Galactic globular cluster (GC) NGC 2419 from two Keck/DEIMOS slitmasks. The probability that there is more than one contaminating halo field star in this sample is extremely low. Analysis of moderate-resolution spectra of these cluster members, as well as of our Keck/HIRES high-resolution spectra of a subsample of them, demonstrates that there is a small but real spread in $\mathrm{Ca}$ abundance of $\sim 0.2$ dex within this massive metal-poor GC. This provides additional support to earlier suggestions that NGC 2419 is the remnant of a dwarf galaxy accreted long ago by the Milky Way.
\end{abstract}

Key words: Galaxy: formation - Galaxy: halo - globular clusters: individual (NGC 2419)

\section{INTRODUCTION}

NGC 2419 is a globular cluster (GC) in the outer halo of the Galaxy at a distance of $84 \mathrm{kpc}^{5}$ It has the highest luminosity $\left(M_{V} \sim-9.6 \mathrm{mag}\right)$ of any $\mathrm{GC}$ with galactocentric radius $R>20 \mathrm{kpc}$, higher than all other GCs with $R>15 \mathrm{kpc}$ with the exception of M54, which is believed to be associated with the nucleus of the Sgr dSph galaxy now being accreted by the Milky Way (Ibata et al. 1995; Sarajedini \& Layden 1995). NGC 2419 also has an unusually large half-light radius $\left(r_{h} \sim 19 \mathrm{pc}\right)$ and core radius for such a massive Galactic GC and a relaxation time which exceeds the Hubble time, also unusual for a GC. Although there is no dynamical evidence for dark matter in NGC 2419 (or any other GC), ${ }^{6}$ if dark matter was initially present in this object, it should not have been depleted from even the central region of this GC. Furthermore, given the position of NGC 2419 in the outer halo, it should not have been affected by any tidal interaction with the Milky Way, assuming its orbit is not extremely eccentric. Baumgardt et al. (2009) determined a velocity dispersion for this cluster based on 40 stars; they found $M / L=2.05 \pm 0.50 M_{\odot} / L_{\odot}$, a normal value for an old stellar system, with no evidence for the presence of dark matter. Harris et al. (1997) obtained a deep color-magnitude diagram (CMD) of NGC 2419 with HST/WFPC2 and demonstrated that there is no detectable difference in age between it and M92, a well studied inner halo GC of comparable metallicity. ${ }^{7}$

Every other luminous GC in the outer halo is considerably more compact. The current version of the online database of Harris (1996) lists only three Galactic GCs that lie anomalously above the bulk of the Galactic GCs in the plane $r_{h}$ versus $M_{V}$, one of which is NGC 2419. The other two are M54 and

\footnotetext{
* Based in part on observations obtained at the W.M. Keck Observatory, which is operated jointly by the California Institute of Technology, the University of California, and the National Aeronautics and Space Administration.

4 Hubble Fellow.

5 This and other parameters for NGC 2419 are from the current version of the online GC database of Harris (1996), based on the photometry of Harris et al. (1997). The first photometric study of this GC was by Racine \& Harris (1975). 6 Although Côté et al. (2002) deduced an apparently high $M / L$ for Pal 13, a low-luminosity distant halo GC, from their measurement of $\sigma_{v} \sim 2.2 \pm 0.4 \mathrm{~km}$ $\mathrm{s}^{-1}$, Siegel et al. (2001) suggest that Pal 13 is "in its final throes of destruction" while Blecha et al. (2004) demonstrate that $\sigma_{v}$ as determined in previous work has been inflated by binaries; with their new measurement there is no need for dark matter.

7 A subsequent analysis of all existing HST data was carried out by Sandquist \& Hess (2008) focusing on the characteristics of the horizontal branch.
}

$\omega$ Cen, the most luminous Galactic GC, and one with a large internal spread in metallicity. The M31 cluster G1 also lies in this anomalous range (Ma et al. 2007). The latter three of these four GCs, all of which are all extremely luminous, are believed to be the stripped cores of dwarf galaxies accreted by our galaxy or, for G1, by M31.

NGC 2419 is quite metal poor. Shetrone et al. (2001) observed one star in this GC with HIRES/Keck; they confirm the low metallicity derived from moderate-resolution spectroscopy, $[\mathrm{Fe} / \mathrm{H}] \sim-2.1$ dex, of Suntzeff et al. (1988). All other existing spectra of stars in NGC 2419 prior to our work are of low signal-to-noise ratio $(\mathrm{S} / \mathrm{N})$, suitable only for radial velocity determination.

Following the early work of Searle \& Zinn (1978) and Zinn (1996), Mackey \& Gilmore (2004) and others have discussed the possibility that some of the outer halo Milky Way GCs have been accreted, while Mackey et al. (2010) argue similarly for the outer halo M31 GCs. Mackey \& van den Bergh (2005) (see also van den Bergh \& Mackey 2004) suggest that NGC 2419 is such a case and that it is the nucleus of a dwarf galaxy that has been accreted by the Milky Way. Casetti-Dinescu et al. (2009) have measured proper motions and determined a preliminary orbit for the Virgo stellar stream; they claim that NGC 2419 is the nucleus of a disrupted stellar system of which the Virgo stellar stream is a part.

There is no evidence from the CMD of multiple stellar populations in NGC 2419, as illustrated in Figure 1. Because of its low metallicity, the isochrones along the red giant branch $(\mathrm{RGB})$ as a function of $[\mathrm{Fe} / \mathrm{H}]$ are very close together in color and thus it will be difficult to detect a star-to-star range in $[\mathrm{Fe} / \mathrm{H}]$ from a measured width in color of the upper giant branch in this cluster's CMD. The observed range in $B-V$ at a fixed $V$ on the upper RGB in the ground-based photometric database of Stetson (2005), on which we rely extensively, in the field of NGC 2419 is $\sim 0.05 \mathrm{mag}$, somewhat larger than the expected observational uncertainty. If this in total is ascribed to metallicity variations, it corresponds to a spread in $[\mathrm{Fe} / \mathrm{H}]$ of $\sim 0.2$ dex. The constraint on potential internal variations of $[\mathrm{Fe} / \mathrm{H}]$ within NGC 2419 set by the width of the upper RGB from both ground and Hubble Space Telescope (HST) observations is comparable as $V-I$ is less sensitive to metallicity variations than is $B-V$.

The existence of multiple populations in GCs has been known to spectroscopists for decades, see, e.g., Cohen et al. (2005) and more recently Carretta et al. (2009). As reviewed 


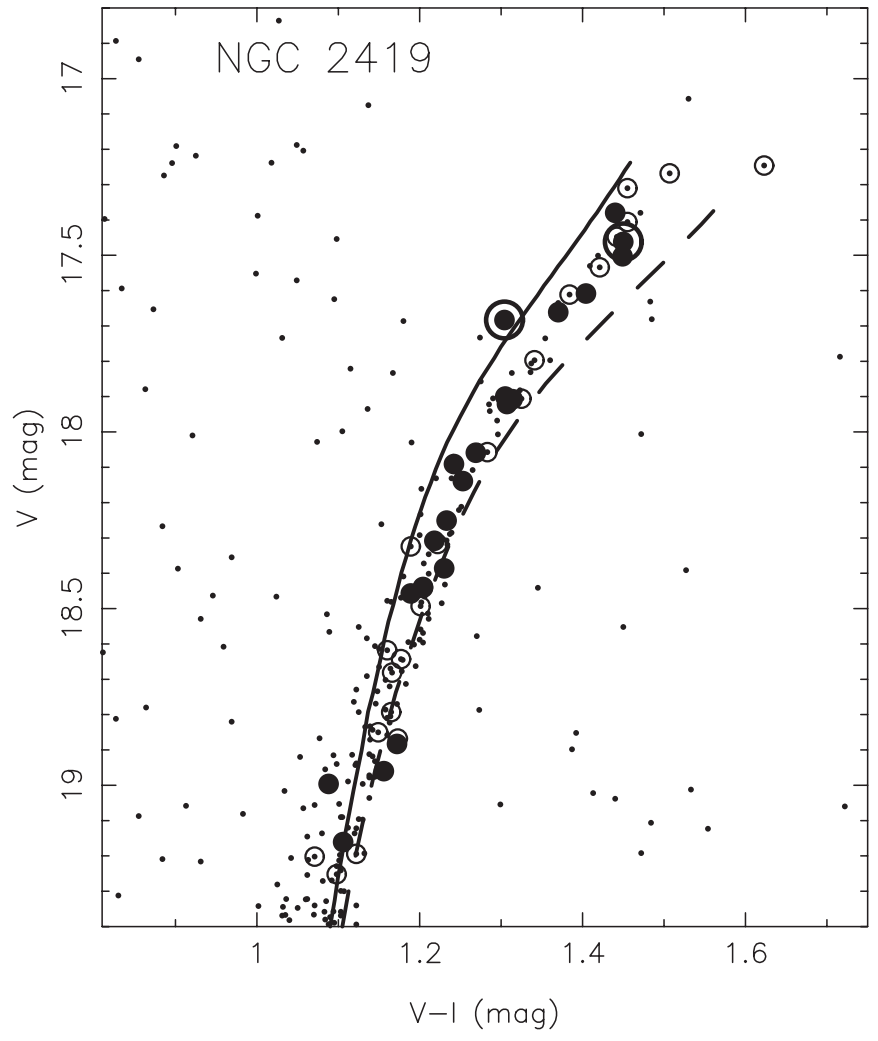

Figure 1. $V, V-I \mathrm{CMD}$ for the database of Stetson (2005) in the field of NGC 2419. Those stars in the DEIMOS slitmasks judged to be members of the GC are indicated by open circles (low CaT) or filled circles (high CaT). The two stars whose membership status is still uncertain are circled. Isochrones from Yi et al. (2003) with $[\alpha / \mathrm{Fe}] 0.3$ dex, age $12 \mathrm{Gyr}$, and $[\mathrm{Fe} / \mathrm{H}]-1.90$ (dashed line) and -2.2 dex (solid line) (interpolated using the code they supply) are shown. A 0.04 mag offset in $V-I$ was applied to both isochrones to improve the fit to the observations.

by Piotto (2009), the exquisite photometry from the HST/ACS Survey of Galactic Globular Clusters (Sarajedini et al. 2007) has enabled the detection of subtle features in GC CMDs that were previously lost in the noise. These include multiple main sequences, a double subgiant branch in NGC 1851 (Milone et al. 2008), and other such phenomena suggesting the presence of multiple populations.

They are explained by small differences in age, He content (which, although difficult to detect directly, must occur in association with the ubiquitous $\mathrm{C} / \mathrm{N}$ and $\mathrm{O} / \mathrm{Na}$ anti-correlations seen in all GCs), and/or heavier metal content. When invoking the latter, one must be careful to distinguish between variations among those light elements that can be produced by ejecta from intermediate- or low-mass asymptotic giant branch (AGB) stars versus variations in the heavier elements such as $\mathrm{Ca}$ or $\mathrm{Fe}$, which are only produced in supernova $(\mathrm{SN})$.

The issue of a second generation of SNII contributions is of particular interest, since it is hard to understand how a GC with little or no dark matter and low binding energy could retain energetic SN ejecta, unless the GC we observe today were a remnant of an initially much more massive stellar system. From both spectroscopy and photometry $\omega$ Cen has been known for more than 30 years to have a wide intrinsic range in $[\mathrm{Ca} / \mathrm{H}]$, $[\mathrm{Fe} / \mathrm{H}]$, etc. extending over a range of $\sim 1.3$ dex with multiple peaks (Norris et al. 1996). The GC M54 also shows this, but it is believed to be part of the central region of the Sgr dSph galaxy, currently being accreted by the Milky Way. Very recently M22, under suspicion for many years, was confirmed to have such star-to-star variations in $\mathrm{Ca} / \mathrm{H}$ and/or $\mathrm{Fe} / \mathrm{H}$ by three independent groups, Da Costa et al. (2009), Marino et al. (2009), and Lee et al. (2009), who each find a range in $[\mathrm{Fe} / \mathrm{H}]$ and/or $[\mathrm{Ca} / \mathrm{H}]$ of $\sim 0.2$ dex. In the present paper, we explore whether NGC 2419 could be another example of a massive GC which has multiple populations with a range in heavy element abundances.

\section{OBSERVATIONS}

We have obtained two multi-object slitmasks of NGC 2419 with the DEIMOS spectrograph (Faber et al. 2003) at the Keck II telescope covering the range $\sim 6500$ to $\sim 9200 \AA$, thus including the near-infrared $\mathrm{Ca}$ triplet. The first of these was used by $\mathrm{J}$. Simon and M. Geha to evaluate their radial velocity accuracy for studies of ultra-faint dwarf spheroidal satellites of the Galaxy (Simon \& Geha 2007). Three $600 \mathrm{~s}$ exposures of this slitmask were taken on 2006 February 2 and three 660 s exposures followed on 2006 February 4. The spectra from each night were summed. Spectra of 65 stars were extracted of which about 35 have adequate $\mathrm{S} / \mathrm{N}$ and appear to be metal-poor red giants which might be members of NGC 2419.

In early 2009, J. Cohen collected from various people (including J. Simon and E. Kirby) spectra from DEIMOS slitmasks of GC fields that include the $8500 \AA$ region. From an initial exploration of such data for six GCs, NGC 2419 was quickly isolated as a potentially interesting case, as even a preliminary analysis suggested there might be a small spread in $\mathrm{Ca}$ abundance based on the strength of the near-IR Ca triplet. Cohen and Kirby then obtained a second DEIMOS slitmask covering NGC 2419, as well as similar data for several other GCs, see Kirby et al. (2010). The second NGC 2419 slitmask was used at the Keck II telescope in 2009 October on two consecutive nights for a total exposure time of $4800 \mathrm{~s}$ and yielded 96 spectra. The position angle of the 2009 slitmask was $178^{\circ}$ different from that of the 2006 slitmask, and the offset between the centers was 42 arcsec. The slitmasks were placed as far as possible from HD 60771, with $V \sim 7.2$ mag, located about 240 arcsec east of the cluster center. Any scattered light from this bright A5 star would appear as a contribution to the diffuse night sky and would be removed via subtraction of the night sky, but inspection of the raw DEIMOS frames showed there is no noticeable background from the star.

Both of these slitmasks were reduced with the DEEP pipeline. ${ }^{8}$ The resulting spectra were then processed through a Figaro (Shortridge 1993) script which determined a continuum level as a function of wavelength, then measured pseudoequivalent widths through bandpasses defined for the two stronger lines in the near-IR Ca triplet at 8542 and $8662 \AA$. These were defined as $\left[1.0-\left(\sum c_{i} / N_{\text {pix }}\right)\right] \Delta \lambda$, where $c_{i}$ is the continuum-normalized DN/pixel, $\Delta \lambda$ is the bandwidth used for the feature, and $N_{\text {pix }}$ is the number of pixels within this bandpass. The weakest triplet line at $8498 \AA$ was ignored due to the low metallicity of the GC of most interest, NGC 2419. DEIMOS slitmasks of eight other Galactic GCs were analyzed in the same way with scripts modified only to take into account the differing radial velocities of each GC and extending the index bandpass widths to accommodate the increasing strength of the Ca triplet lines for more metal-rich GCs.

The two slitmasks for NGC 2419 combined yielded moderateresolution spectra of more than 130 stars. Those which upon visual inspection were not metal-poor giants, including stars appearing too metal-rich, $\mathrm{M}$ dwarfs, or stars with strong Paschen

\footnotetext{
8 See http://astro.berkeley.edu/ cooper/deep/spec2d
} 


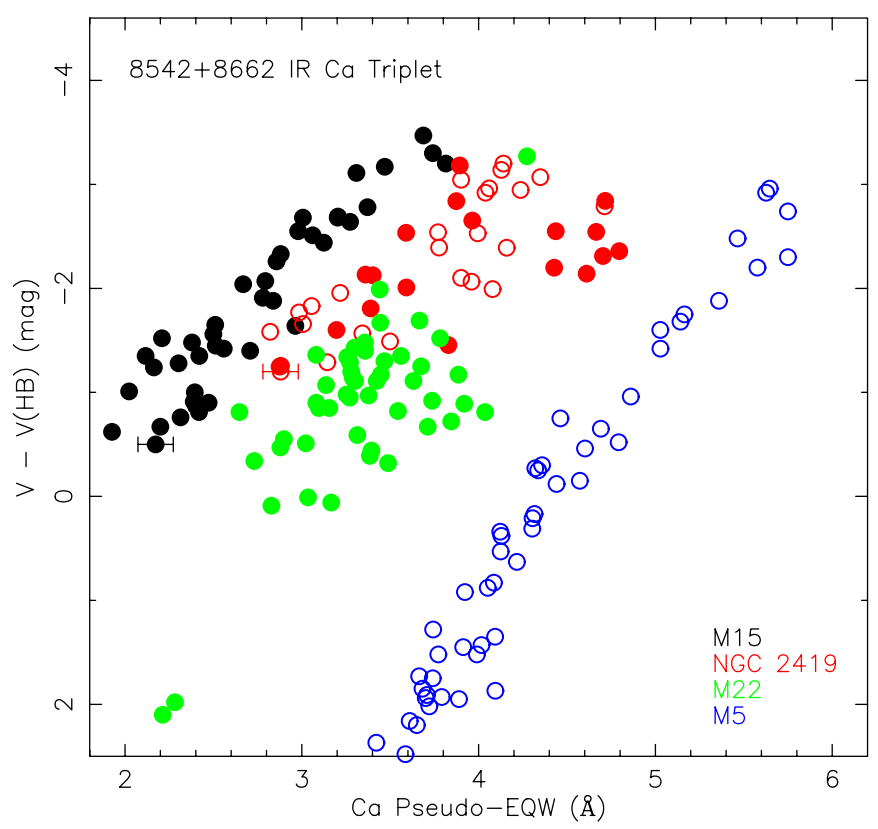

Figure 2. Sum of the pseudo-equivalent widths for the two stronger lines of the near-infrared $\mathrm{Ca}$ triplet are shown as a function of $M_{V}$ corrected for extinction and normalized with respect to the location of the horizontal branch for four globular clusters (M15, NGC 2419, M22, and M5, in order of increasing metallicity), each shown as a different color. The NGC 2419 apparent members, shown in red, are filled circles if the star was on both DEIMOS slitmasks and open circles if included in only one of the two slitmasks. The two stars whose membership in NGC 2419 is still uncertain are not shown. Non-members have been carefully eliminated for NGC 2419 only. As discussed in Section 1, the GC M22, which like NGC 2419, also shows a significant spread in this figure, is known to have a small internal range in $[\mathrm{Ca} / \mathrm{H}]$.

lines, were rejected. In addition, those which were discrepant from the cluster mean $v_{r}$ by more than $15 \mathrm{~km} \mathrm{~s}^{-1}$ as determined from cross correlations were rejected. A further requirement imposed was that a candidate member be within 0.1 mag of the $V, V-I$ locus of the RGB of NGC 2419 as defined from the photometric database of Stetson (2005). This left a sample of 45 apparent RGB members of NGC 2419. Figure 2 shows the sum of the pseudo-equivalent widths for the two stronger Ca triplet lines, which we denote CaT, measured in this way from the two slitmasks of NGC 2419, with two possible non-members omitted (43 RGB stars in total), together with large samples from the well-studied Galactic GCs M15, M22, and M5. Note the apparent spread in CaT at a fixed $V$ mag of the NGC 2419 sample, also shown by the M22 sample, but not by the M15 or M5 giants.

The CaT measurements for the 43 NGC 2419 members isolated from our DEIMOS slitmasks have been converted into $[\mathrm{Ca} / \mathrm{H}]$ values and their histogram is shown in Figure 3. In this exercise, the calibrating GCs we used are M15, M53, and $\mathrm{M} 13$, with $[\mathrm{Fe} / \mathrm{H}]$ assumed to be $-2.26,-1.99$, and -1.54 dex, respectively, adopted from the online catalog of Harris (1996). Furthermore, based on the cumulative result of the many studies of GCs reviewed and summarized by Gratton et al. (2004), we assume that $[\mathrm{Ca} / \mathrm{Fe}]$ is constant at +0.25 dex for all of the red giants in the calibrating GCs. The sensitivity of $\mathrm{Ca}$ triplet line strength to $[\mathrm{Ca} / \mathrm{H}]$ is strong in this low-metallicity regime $([\mathrm{Fe} / \mathrm{H}] \lesssim-2$ dex $)$; CaT changes by $0.1 \AA$ for a 0.02 dex change in $[\mathrm{Ca} / \mathrm{H}]$. Figure 3 shows that $[\mathrm{Ca} / \mathrm{H}](\mathrm{CaT})$ in NGC 2419 is strongly peaked at about -1.9 dex, with a tail of members extending to somewhat higher metallicity. The exact peak metallicity for NGC 2419 depends

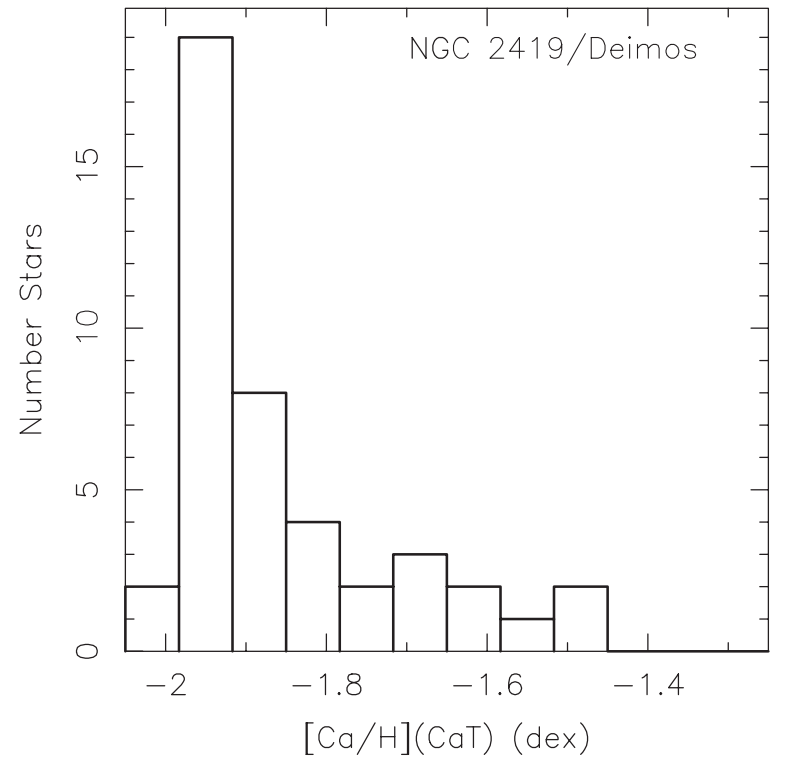

Figure 3. Histogram of $[\mathrm{Ca} / \mathrm{H}]$ inferred from their $\mathrm{CaT}$ for the 43 members of NGC 2419. The calibrating GCs are M15, M53, and M13, with [Fe/H] assumed to be $-2.26,-1.99$, and -1.54 dex respectively, adopted from the online catalog of Harris (1996). A second order fit to our DEIMOS spectra of samples of stars in each of the three calibrators as a function of $V-V(\mathrm{HB})$ is defined. Then, for each of the NGC 2419 members, another second order fit to [Fe/H] as a function of CaT for the three calibrators at the luminosity $V-V(\mathrm{HB})$ of the NGC 2419 star yields its $[\mathrm{Fe} / \mathrm{H}] .[\mathrm{Ca} / \mathrm{Fe}]$ is assumed to be constant at +0.25 dex for all red giants in the calibrating GCs.

on the $[\mathrm{Fe} / \mathrm{H}]$ values assigned to the calibrating GCs and on the constant $[\mathrm{Ca} / \mathrm{Fe}]$ adopted. The latest detailed abundance analysis for M15 luminous giants by Carretta et al. (2009) finds $[\mathrm{Fe} / \mathrm{H}]-2.32$ dex, 0.06 dex below the value adopted here; their somewhat lower $[\mathrm{Fe} / \mathrm{H}]$ for this $\mathrm{GC}$ is supported by as yet unpublished work by J. Cohen and J. Melendez. M53 is the least well studied at high dispersion of the calibrators and thus might have a value for $[\mathrm{Fe} / \mathrm{H}]$ in the Harris (1996) database which is incorrect by up to \pm 0.15 dex.

Even from just the 2006 slitmask, it was clear that NGC 2419 showed signs of having a spread in $\mathrm{Ca} / \mathrm{H}$. A campaign was therefore launched to obtain high-resolution spectra of a number of RGB stars in this GC, with eight stars observed with HIRES (Vogt et al. 1994) at the Keck I telescope, of which five have reasonable $\mathrm{S} / \mathrm{N}$, while the other three, observed with HIRES-B during a run for another program, are somewhat lower in $\mathrm{S} / \mathrm{N}$. In addition, several somewhat fainter stars suspected to have unusually strong $\mathrm{Ca}$ triplet lines from the moderate-resolution DEIMOS spectra were observed with HIRES in 2010 February with typical exposures of $1800 \mathrm{~s}$ to get accurate radial velocities.

The HIRES spectra were analyzed in a manner similar to that described in Cohen et al. (2008) with one major difference intended to improve the relative accuracy of the determination of $T_{\text {eff }}$ for each star; we explicitly assumed that the NGC 2419 RGB stars all lie on a single isochrone. Full details of the abundance analysis will be given in J.G. Cohen et al. (2010, in preparation). Figure 4 compares the resulting derived $[\mathrm{Fe} / \mathrm{H}]$ and $[\mathrm{Ca} / \mathrm{H}]$ for each of the 10 candidate members of NGC 2419 with detailed abundance analyses to their $[\mathrm{Ca} / \mathrm{H}]$ as measured from the DEIMOS slitmasks. It is clear that the $[\mathrm{Ca} / \mathrm{H}] \mathrm{CaT}$ (from DEIMOS spectra) are correlated with the $[\mathrm{Ca} / \mathrm{H}]$ from the HIRES spectra and that both data sets suggest that a small range in $\mathrm{Ca}$ abundance exists among the upper RGB stars in NGC 2419. [Ca/Fe] for the eight members with HIRES spectra 


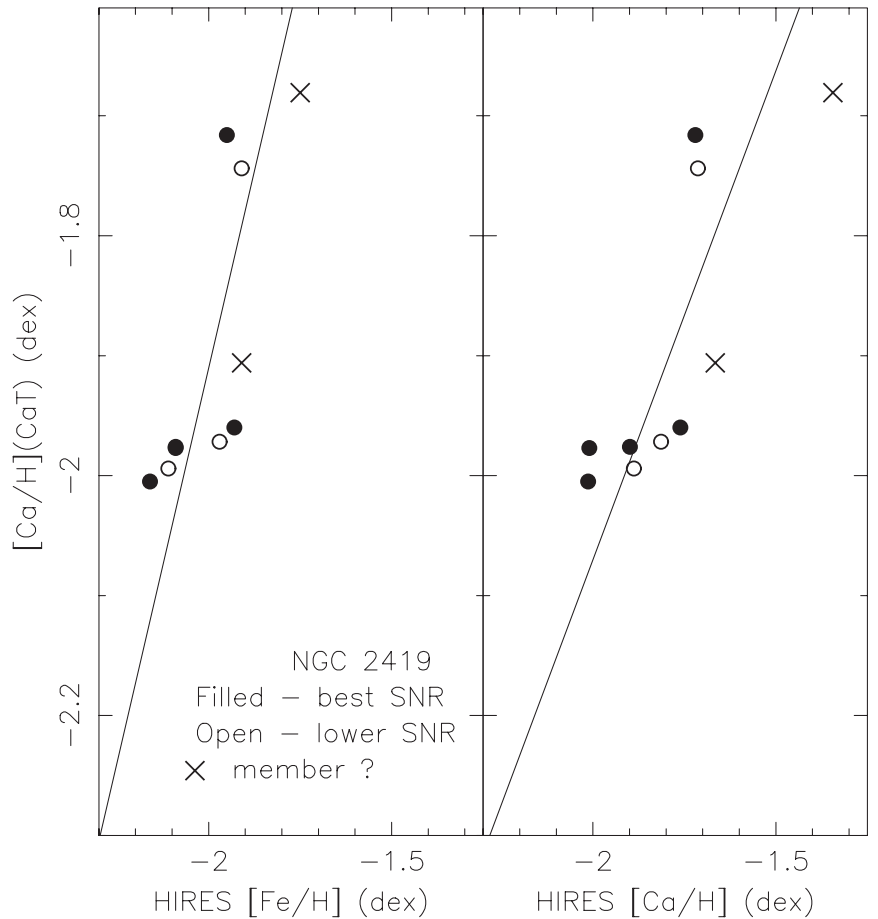

Figure 4. $[\mathrm{Ca} / \mathrm{H}]$ derived from the sum of the pseudo-equivalent widths for the two stronger lines of the near-infrared $\mathrm{Ca}$ triplet are shown as a function of the $[\mathrm{Fe} / \mathrm{H}]$ (left panel) or $[\mathrm{Ca} / \mathrm{H}]$ (right panel) for the NGC 2419 stars with HIRES spectra. Giants with higher S/N spectra are indicated as filled circles; open circles denote the members with lower $\mathrm{S} / \mathrm{N}$ spectra, two of which, marked by crosses, still have concerns regarding membership (see Section 3).

is +0.17 dex with $\sigma=0.05$ dex; the total range in $[\mathrm{Fe} / \mathrm{H}]$ and $[\mathrm{Ca} / \mathrm{H}]$ is 0.25 and 0.31 dex, respectively, which ranges are identical to within the errors.

\section{MEMBERSHIP}

Establishing the membership of candidates in NGC 2419 is complicated as the cluster mean $v_{r}$ is $\sim-20.3 \mathrm{~km} \mathrm{~s}^{-1}$, which is not very far from the mean of the halo field at about $0 \mathrm{~km}$ $\mathrm{s}^{-1}$. Furthermore, the metallicity of NGC 2419 is close to that of the mean of field stars with kinematics suggesting their orbits reach the outer halo of $[\mathrm{Fe} / \mathrm{H}] \sim-2.0 \mathrm{dex}$ (Carollo et al. 2007), so confusion with any halo field giants whose distances are comparable to that of the GC may occur. Moderate-resolution spectra can rule out only those stars which have $v_{r}$ or CaT considerably different from the cluster mean. The radial velocity uncertainty from the DEIMOS spectra for NGC 2419 stars in the relevant magnitude range was determined by comparing the $v_{r}$ measured from each of the two slitmasks, taken three years apart, for the 19 stars in common. The result was a difference in the mean of 0.01 pixels with $\sigma$ less than 0.2 pixels on the detector; this corresponds to $\sigma \sim 2.3 \mathrm{~km} \mathrm{~s}^{-1}$.

With regard to the initial list of 10 discrepant stars with CaT somewhat high for their $V$ from Figure 2, three were already confirmed as members from the high-resolution spectra of Olszewski et al. (1993) or Baumgardt et al. (2009). All but one of the remaining seven were observed with short exposures with HIRES in 2010 February. Of these, the status of NGC 2419 S951 remains unclear. Even though it lies on the cluster locus in the CMD, and has a spectrum which appears reasonable for a member, its $v_{r}$ is $15 \mathrm{~km} \mathrm{~s}^{-1}$ lower than the cluster mean $v_{r}$; the velocity dispersion in this GC is about $4 \mathrm{~km} \mathrm{~s}^{-1}$ (Baumgardt et al. 2009). This star could be a binary in this GC,

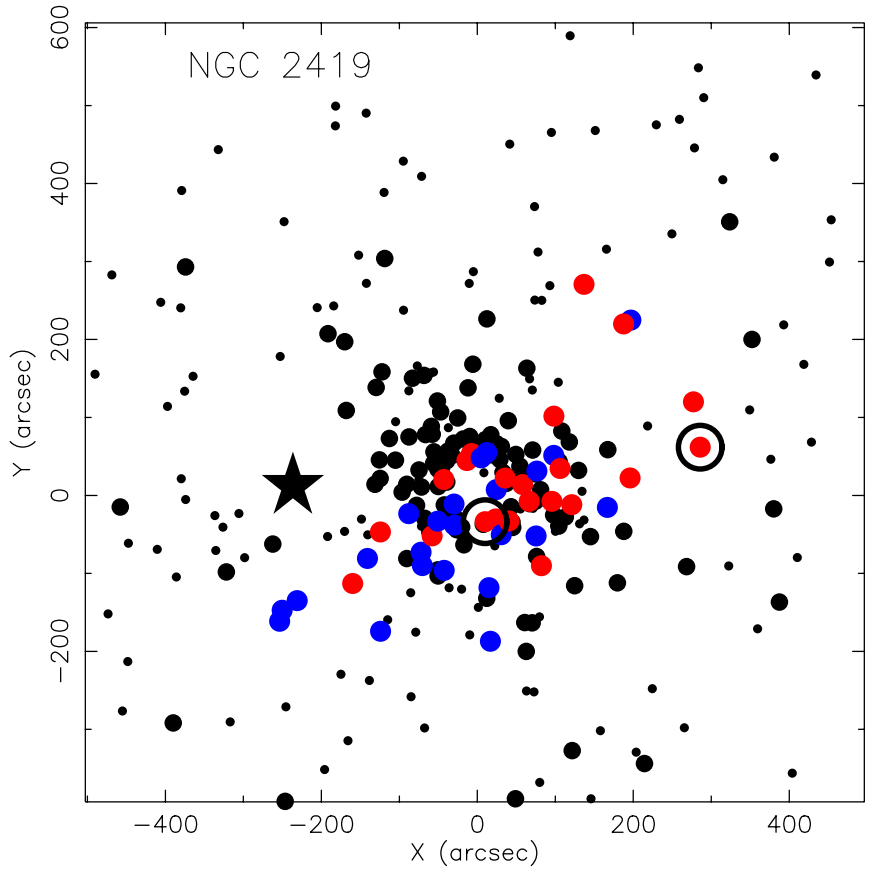

Figure 5. Positions on the sky of the stars from the photometric database of Stetson (2005) are indicated by small black dots. Those with $17.2<V<19.2$ mag and within $0.2 \mathrm{mag}$ of the NGC 2419 locus in the $V, V-I$ CMD are shown by larger filled circles. Objects in the DEIMOS slitmasks with low CaT (blue circles) and those with higher $\mathrm{CaT}$ (red circles) for their $V$ mag are indicated. The two circled red points are apparent members whose membership is still uncertain (see Section 3); NGC 2419 S951 is the one close to the center of the cluster, while S1673 is far from the center. The position of HD 60771 is indicated by a large star.

but the two spectra taken three years apart have the same $v_{r}$ to within the uncertainties, or it could simply be far in the tail of the $v_{r}$ distribution. NGC 2419 S1673 has $v_{r}$ consistent with membership in this GC, but it lies 0.06 mag bluer than the RGB locus for this GC. It also has slightly higher $[\mathrm{Ca} / \mathrm{H}]$ and $[\mathrm{Fe} / \mathrm{H}]$ derived from its HIRES spectrum than the eight stars we judge members of this GC, as shown in Figure 4. We suspect that it may be an AGB member, as it, together with the most luminous NGC 2419 RGB stars, shows strong emission in both the red and blue wings of $\mathrm{H} \alpha$. Ignoring these two stars leaves a sample of 43 RGB stars expected to be members of NGC 2419.

Figure 5 shows the region of NGC 2419 on the sky with the positions of the stars in our sample indicated as well as those of the entire Stetson photometric database in this field and that of HD 60771. This figure suggests that the field star contamination of our NGC 2419 sample of upper RGB stars should be small. To quantify this, we first consider the observed star counts in a region on the sky within the Stetson photometric database for NGC 2419 but far from the cluster center. Within an area of $69 \operatorname{arcmin}^{2}$, there are no stars which lie within $0.1 \mathrm{mag}$ of the NGC 2419 locus in the $V, V-I$ CMD with $17.2<V<19.2 \mathrm{mag}$. This yields a maximum background rate of 0.01 field stars $\operatorname{arcmin}^{-} 2$. Since there is considerable overlap in the area of the two DEIMOS slitmasks, we set the total area we used to $100 \mathrm{arcmin}^{2}$ to predict a maximum of 1 field star. We then require that $v_{r}$ must be that of the mean for this GC to within $10 \mathrm{~km} \mathrm{~s}^{-1}$; NGC 2419 S951, whose membership status is still uncertain as discussed above, is the only star between 10 and $15 \mathrm{~km} \mathrm{~s}^{-1}$ off the cluster mean that survived the initial series of checks. We adopt the values for the "outer halo" of Carollo et al. (2010) of $\left\langle v_{R}\right\rangle=-8.6 \pm 6.1 \mathrm{~km} \mathrm{~s}^{-1}, \sigma\left(v_{R}\right)=159 \pm 4 \mathrm{~km} \mathrm{~s}^{-1}$, 
noting that these apply to a region at much smaller galactocentric radius than the one under consideration here. Then, our criterion for $v_{r}$ (assumed equal to $v_{r}$, where $R$ is the galactocentric radius) rejects $95 \%$ of the field halo stars. This cuts the expected number of field stars in our sample to less than 0.005 field stars arcmin ${ }^{-} 2$. Figure 5 also suggests there may be a spatial asymmetry in the distribution of the high CaT versus the low CaT RGB stars in NGC 2419.

The expected field star contamination was also evaluated using the Besançon model of the Milky Way (Robin et al. 2003). With the rough color selection used to design the 2006 slitmask (which included a somewhat broader box than just within 0.1 mag of the cluster RGB), the surface density of halo giants meeting the velocity cut of $-35 \mathrm{~km} \mathrm{~s}^{-1}<v_{r}<-5 \mathrm{~km} \mathrm{~s}^{-1}$ is predicted to be to be $\sim 0.001$ stars $\operatorname{arcmin}^{-} 2$. Given the radius for the most distant RGB candidate member of NGC 2419 of $500^{\prime \prime}$, the expected number of halo giants in the observed area is $\sim 0.2$. One could then apply an additional metallicity cut to select only stars within some range around the cluster $[\mathrm{Fe} / \mathrm{H}]$ value, but we conservatively assume that all of the velocity and color-selected stars will also lie in the right metallicity range. One must recognize that the Besançon model for the outer halo is probably not well calibrated at such large galactocentric radii. Furthermore, it does not have substructure, which is almost certainly dominant at large radii.

Since both of these tests predict a number of field star interlopers in our carefully selected sample of less than one, we conclude that the large population in the DEIMOS sample in the field of NGC 2419 of RGB stars with somewhat higher CaT must almost entirely be members of this GC.

\section{ACCURACY OF CAT}

We next demonstrate that the range in $\mathrm{CaT}$ at a fixed $V$ near the tip of the RGB shown in Figure 4 is real. NGC 2419 is the most distant GC shown in this figure, but the M5 sample extends more than 5 mag below the RGB tip of this GC, comparable to the difference in distance modulus between M5 and NGC 2419. With only a minimal effort to remove non-members, the M5 sequence is much tighter even at the faintest levels probed. The bulk of the M22 sample is also several magnitudes below the RGB tip, but does not reach as faint as the NGC 2419 giants.

The DEIMOS spectra have a resolution $\lambda / \Delta \lambda \sim 7000$ with $0.32 \AA$ pixel $^{-1}$ near $8500 \AA$. Our criterion for a suitable $S / N$ DEIMOS spectrum for a candidate member of NGC 2419 is a total of 3000 detected electrons/pixel in the continuum at $8600 \AA$. Thus, ignoring issues of sky subtraction of night sky emission lines, which fortunately are few and fairly weak in the region of the near-infrared Ca triplet, the $\mathrm{S} / \mathrm{N}$ per each $1.3 \AA$ spectral resolution element in the continuum at $8600 \AA$ will exceed 110 as each of the two slitmasks has a total exposure time of more than $1 \mathrm{hr}$.

We used a width of $25 \AA$ for the $8542 \AA$ line index and of $20 \AA$ for the weaker $8662 \AA$ line index for candidate members of NGC 2419, for a total bandpass for the CaT measurement of $45 \AA$, which is $\sim 34$ spectral resolution elements for the two Ca II lines. A total bandpass of $350 \AA$ was used in the continuum fitting, corresponding to 290 spectral resolution elements.

The range in CaT at a fixed $V$ near the RGB tip in NGC 2419 is about $1 \AA$. Thus, a $2.2 \%$ change in the continuum level across the entire $\mathrm{CaT}$ region could produce the observed width. However, our spectra were analyzed with scripts in a fully automatic manner. No manual intervention occurred. Given the $\mathrm{S} / \mathrm{N}$ requirement for the DEIMOS spectra described above, it is difficult to understand how an error in the continuum location systematically either high or low at the level required could have occurred. Beyond the issue of scattered light from the nearby bright star HD 60771, discussed and dismissed earlier in Section 2, we know of no other issues that might introduce an apparent spread for just NGC 2419 among our sample of Galactic GCs with DEIMOS slitmasks.

But we have more quantitative demonstrations of the accuracy of our CaT measurements for NGC 2419. There are two slitmasks in this GC taken three years apart, with considerable overlap between them. Also, the two sequences of exposures taken two nights apart of the 2006 slitmask were each reduced independently. The CaT values were measured from each, then averaged. The mean $(\sigma)$ of the differences between $\mathrm{CaT}$ from the first exposure and that from the second is $0.08 \AA(0.13 \AA)$ for the 27 stars from the 2006 DEIMOS slitmask which were accepted as members of NGC 2419 with $V<19.3 \mathrm{mag}$. Considering the 23 stars brighter than $V=18.8 \mathrm{mag}$, this is reduced to $0.05 \AA$ with $\sigma=0.11 \AA$.

There are 16 stars in common between the two slitmasks. The mean of the differences between CaT from each of the two slitmasks is $0.03 \AA$ with $\sigma=0.13 \AA$. This again demonstrates that the spread seen in Figure 2 for the NGC 2419 red giants is real. The adopted CaT values are averages of those from the 2006 and the 2009 slitmask, for those stars included in both slitmasks, and are given in Table 1.

\subsection{Comparison with Spectral Synthesis of the DEIMOS Spectra}

Kirby et al. (2008) and Kirby et al. (2010) have measured $[\mathrm{Fe} / \mathrm{H}]$ and $[\mathrm{Ca} / \mathrm{Fe}]$ abundances for stars in Galactic GCs and dwarf spheroidal galaxies. NGC 2419 was among their sample. In fact, they used the same DEIMOS data from which we measure $\mathrm{Ca}$ triplet metallicities. Because their technique is based on spectral synthesis and because the $\mathrm{Ca}$ triplet lines are excluded from their analysis, their measurements are independent of our $\mathrm{Ca}$ triplet-based results. Figure 6 shows the spectral synthesisbased $[\mathrm{Fe} / \mathrm{H}]$ and $[\mathrm{Ca} / \mathrm{H}]$ distribution functions, where $[\mathrm{Ca} / \mathrm{H}]$ is the sum of $[\mathrm{Fe} / \mathrm{H}]$ and $[\mathrm{Ca} / \mathrm{Fe}]$ from the set of 43 stars isolated as probable members of NGC 2419 here (see Table 1) and with $[\mathrm{Fe} / \mathrm{H}]$ and $[\mathrm{Ca} / \mathrm{Fe}]$ errors less than $0.2 \mathrm{dex} ; 38$ of the 43 meet all these criteria. The spectral synthesis-based $[\mathrm{Ca} / \mathrm{H}]$ distribution is asymmetric in the same sense as is that derived from CaT: a peak followed by a tail toward higher $\mathrm{Ca}$ abundances, as can be seen by comparing Figure 6 from the spectral syntheses versus Figure 3 from the $\mathrm{Ca}$ triplet analysis. A comparison for each individual star in NGC 2419 from our sample that meets these criteria is shown in Figure 7. The dashed line in this figure indicates equality between the $\mathrm{Ca}$ abundances $[\mathrm{Ca} / \mathrm{H}]$ derived from the $\mathrm{Ca}$ triplet lines and that from spectral synthesis and strongly supports the reality of the small range in $\mathrm{Ca}$ abundance we claim to exist in NGC 2419.

Curiously, the $[\mathrm{Fe} / \mathrm{H}]$ distribution for NGC 2419 derived from spectral synthesis of the DEIMOS data is not as broad as the $[\mathrm{Ca} / \mathrm{H}]$ distribution. The $[\mathrm{Ca} / \mathrm{H}]$ measurements are slightly more uncertain than the $[\mathrm{Fe} / \mathrm{H}]$ measurements because the DEIMOS spectra contain fewer $\mathrm{Ca}$ lines than Fe lines. Figure 6 also includes the $[\mathrm{Fe} / \mathrm{H}]$ and $[\mathrm{Ca} / \mathrm{H}]$ distributions for $\mathrm{M} 13$, a GC known not to have a measurable dispersion in elements heavier than Mg (see, e.g., Sneden et al. 2004; Cohen \& Melendez 2005). The $[\mathrm{Ca} / \mathrm{H}]$ distribution in M13 is slightly broader than its $[\mathrm{Fe} / \mathrm{H}]$ distribution, but not as broad as the $[\mathrm{Ca} / \mathrm{H}]$ distribution in NGC 2419. These spectra for these two clusters were obtained 
Table 1

Data for Apparent Red Giant Members of NGC 2419

\begin{tabular}{|c|c|c|c|c|}
\hline Name $^{a}$ & $\begin{array}{c}V^{\mathrm{a}} \\
(\mathrm{mag})\end{array}$ & $\begin{array}{l}\mathrm{CaT} \\
(\AA) \\
\end{array}$ & $\begin{array}{c}\text { Detected Electrons } \\
\text { Per Pixel }^{\text {b }} \\
\end{array}$ & Masks ${ }^{c}$ \\
\hline D6 & 18.457 & 4.08 & 6040 & 1 \\
\hline D17 & 18.681 & 2.98 & 6555 & 1 \\
\hline D18 & 17.921 & 4.00 & 10000 & 1 \\
\hline S217 & 19.250 & 2.88 & 3080 & 1 \\
\hline $\mathrm{S} 223$ & 17.250 & 4.14 & 34050 & 1 \\
\hline $\mathrm{S} 243$ & 19.202 & 2.87 & 12010 & 2 \\
\hline S357 & 18.996 & 3.83 & 13270 & 2 \\
\hline S406 & 17.797 & 3.96 & 61015 & 2 \\
\hline S457 & 18.317 & 3.36 & 34050 & 2 \\
\hline $\mathrm{S} 458$ & 17.900 & 4.44 & 52378 & 2 \\
\hline S642 & 18.493 & 3.22 & 5945 & 1 \\
\hline S656 & 18.620 & 3.06 & 6195 & 1 \\
\hline S704 & 18.308 & 4.61 & 33265 & 2 \\
\hline S731 & 18.793 & 3.01 & 4185 & 1 \\
\hline S759 & 18.883 & 3.34 & 3500 & 1 \\
\hline S809 & 17.915 & 3.59 & 51800 & 2 \\
\hline S810 & 17.310 & 4.13 & 28200 & 1 \\
\hline $\mathrm{S} 870$ & 18.059 & 4.16 & 6310 & 1 \\
\hline S890 & 18.960 & 3.50 & 3410 & 1 \\
\hline S934 & 18.324 & 3.40 & 29870 & 2 \\
\hline S957 & 17.530 & 4.04 & 21400 & 1 \\
\hline S973 & 17.488 & 4.06 & 19380 & 1 \\
\hline S1004 & 17.907 & 4.66 & 53380 & 2 \\
\hline S1008 & 17.910 & 3.78 & 13660 & 1 \\
\hline S1029 & 18.057 & 3.78 & 9430 & 1 \\
\hline S1048 & 17.380 & 4.35 & 19940 & 1 \\
\hline S1065 & 17.660 & 4.71 & 18080 & 1 \\
\hline S1131 & 17.608 & 4.72 & 78300 & 2 \\
\hline S1166 & 17.503 & 4.24 & 19130 & 1 \\
\hline S1209 & 17.406 & 3.90 & 20730 & 1 \\
\hline S1215 & 18.850 & 3.20 & 18470 & 2 \\
\hline S1245 & 18.386 & 3.96 & 6180 & 1 \\
\hline S1294 & 18.139 & 4.70 & 39580 & 2 \\
\hline S1305 & 17.611 & 3.87 & 74800 & 2 \\
\hline S1306 & 18.091 & 4.80 & 36840 & 2 \\
\hline S1340 & 19.161 & 3.14 & 2870 & 1 \\
\hline S1391 & 18.441 & 3.59 & 28760 & 2 \\
\hline S1497 & 18.643 & 3.39 & 22240 & 2 \\
\hline S1563 & 18.349 & 3.90 & 6225 & 1 \\
\hline S1664 & 18.251 & 4.43 & 34800 & 2 \\
\hline S1810 & 19.194 & 2.88 & 2885 & 1 \\
\hline S1814 & 17.268 & 3.89 & 117100 & 2 \\
\hline S1819 & 18.868 & 2.82 & 4000 & 1 \\
\hline S951 ${ }^{\mathrm{d}}$ & 17.270 & 4.45 & 90860 & 2 \\
\hline $\mathrm{S} 1673^{\mathrm{d}}$ & 17.683 & 4.73 & 61010 & 2 \\
\hline
\end{tabular}

Notes.

a Star IDs and $V$ mags are from the database of Stetson (2005).

$\mathrm{b}$ Total detected electrons/pixel for the two DEIMOS slitmasks in the continuum near $8600 \AA$ A. At $8500 \AA$, 1 pixel $\equiv 0.32 \AA$.

c 1 : star in one of two slitmasks; 2: star in both.

d These two stars may be members of of NGC 2419; see Section 3.

and the abundances were measured via spectral synthesis using the same techniques. Therefore, based on spectral synthesis we conclude that the $[\mathrm{Ca} / \mathrm{H}]$ distribution in NGC 2419 has a measurable dispersion whereas the dispersion in $[\mathrm{Fe} / \mathrm{H}]$ is not obvious. In this context, one should note the larger range and hence shallower slope (0.76 versus 1.23$)$ in Figure 4 for $[\mathrm{Ca} / \mathrm{H}]$ than for $[\mathrm{Fe} / \mathrm{H}]$ found from the much smaller set of stars with HIRES spectra. This issue will be discussed in more detail in a future publication.

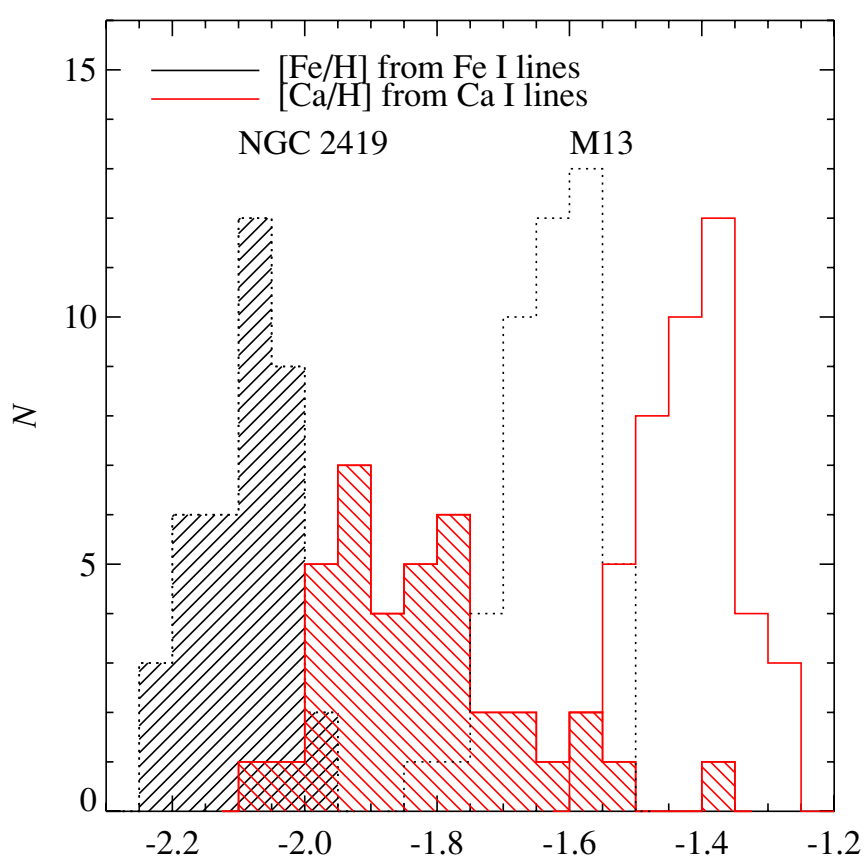

$[\mathrm{Fe} / \mathrm{H}]$ or $[\mathrm{Ca} / \mathrm{H}]$ from spectral synthesis

Figure 6. Spectral synthesis-based $[\mathrm{Fe} / \mathrm{H}]$ (black) and $[\mathrm{Ca} / \mathrm{H}]$ (red) distributions for stars in the globular clusters NGC 2419 (shaded) and M13 (open). Only stars from the list of probable members of this GC isolated here (see Table 1) with estimated uncertainties of less than $0.2 \mathrm{dex}$ in both quantities are included.

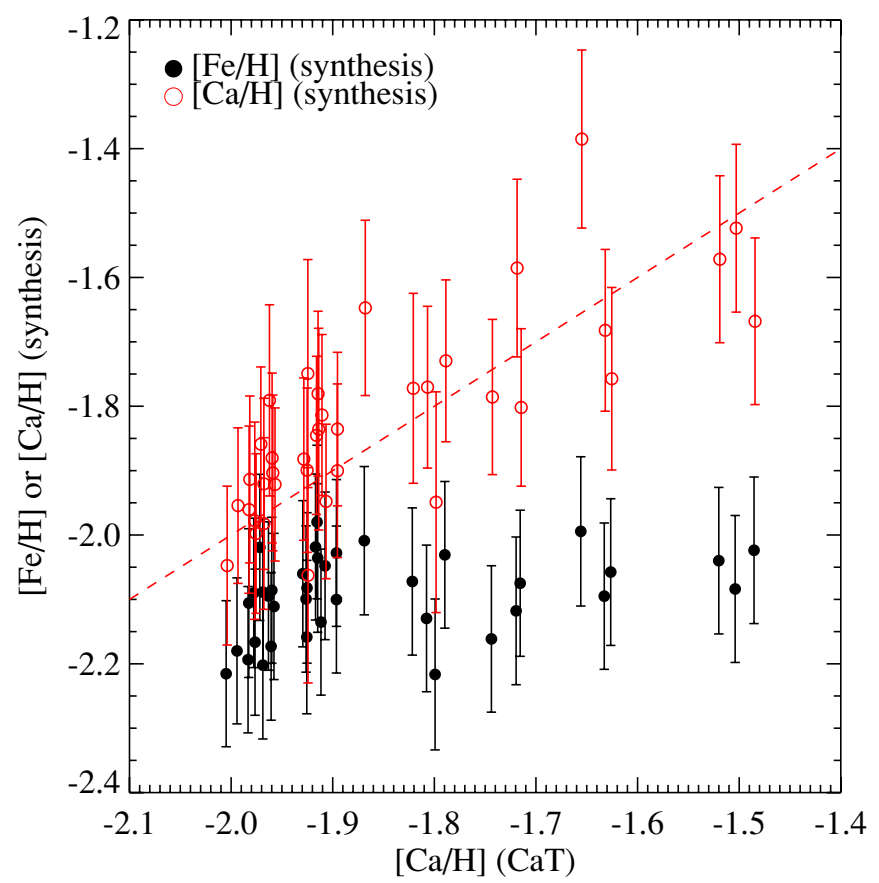

Figure 7. Spectral synthesis-based $[\mathrm{Fe} / \mathrm{H}]$ (black) and $[\mathrm{Ca} / \mathrm{H}]$ (red) for stars in NGC 2419 are shown as a function of $[\mathrm{Ca} / \mathrm{H}](\mathrm{CaT})$. Only stars from the list of probable members of NGC 2419 isolated here (see Table 1) with estimated uncertainties of less than 0.2 dex in both quantities are included. The dashed line indicates equality between $[\mathrm{Ca} / \mathrm{H}](\mathrm{CaT})$ derived from the $\mathrm{Ca}$ triplet lines and that from spectral synthesis excluding the triplet lines.

\section{DISCUSSION}

We have isolated a sample of 43 upper RGB stars in the massive Galactic GC NGC 2419, located far out in the halo of the Milky Way at a distance of $84 \mathrm{kpc}$. This sample must consist almost entirely of members of NGC 2419, as multiple 
lines of evidence demonstrate that the expected number of halo field stars which could meet our stringent requirements for membership and lie within the area covered by our DEIMOS slitmasks is less than one. Analysis of two DEIMOS slitmasks taken three years apart at the Keck II telescope in the field of NGC 2419 demonstrates that these cluster members show a small but real range in the strength of their near-IR Ca II triplet lines at a fixed $V$ mag (see Figure 2). We emphasize that we have such data in hand for $\sim 10$ Galactic GCs and that ignoring M22, NGC 2419 is the most credible case for which star-to-star variations of $\mathrm{Ca}$ abundances were seen.

The range in NGC 2419 of CaT (the sum of the pseudoequivalent width of the two stronger lines of the near-infrared Ca triplet) at a fixed $V$ mag near the tip of the RGB is $\sim 1 \AA$, from $\sim 3.8$ to $\sim 4.8 \AA$. If we assume the two Ca II lines we use, at 8542 and $8662 \AA$, are on the damping part of the curve of growth, then the $\mathrm{Ca} / \mathrm{H} \propto(\mathrm{CaT})^{2}$, equivalent to a range of $0.2 \mathrm{dex}$ in $[\mathrm{Ca} / \mathrm{H}]$. That is about the range seen in $[\mathrm{Ca} / \mathrm{H}]$ in detailed abundance analyses of high-resolution spectra of eight members of this cluster that we have also completed. The $[\mathrm{Ca} / \mathrm{H}]$ histogram for our sample of NGC 2419 giants deduced from our CaT measurements as calibrated by DEIMOS slitmasks for M15, M53, and M5 (Figure 3) shows a strong peak in $[\mathrm{Ca} / \mathrm{H}](\mathrm{CaT})$ at about -1.9 dex with a tail extending to higher metallicities. This result is supported by a spectral synthesis analysis of the same DEIMOS slitmask following the techniques developed in Kirby et al. $(2008,2010)$ which specifically excludes the $\mathrm{Ca}$ triplet lines. We therefore assume that the range in $[\mathrm{Ca} / \mathrm{H}]$ in NGC 2419 is real. A careful examination of the existing DEIMOS slitmasks in GC fields, and of those soon to be acquired, for star-to-star variations in $\mathrm{Ca}$ abundance thus seems well justified and is underway.

While many clusters show an intrinsic dispersion in lighter elements up to and including $\mathrm{Al}$ (see, e.g., Carretta et al. 2009), very few show a measurable dispersion in heavier elements. Dispersions in lighter elements could be explained by selfpollution by AGB star winds, but spreads in $\mathrm{Ca}$ abundance must arise from enrichment by SNe. Based on the half-light radius in the Harris catalog and the mass given by Baumgardt et al. (2009), the gravitational binding energy of NGC 2419 is about $4 \times 10^{51} \mathrm{erg}$. A few core-collapse SNe would have been energetic enough to evacuate the cluster of gas if NGC 2419 formed in isolation. The system in which NGC 2419 formed must have been massive enough to retain the SN ejecta. We suggest that NGC 2419 formed in a now-disrupted dwarf galaxy. The recent theoretical model of GC formation by Conroy \& Spergel (2010) follows gas flows into and out of the young cluster in detail and can reproduce many features of the chemical inventory of GCs arising from multiple stellar generations, specifically the correlated and anti-correlated variations among the light elements and the relative fractions of these generations as observed today (see, e.g., Cohen et al. 2005; Carretta et al. 2009). However, even this model cannot accommodate a spread in heavy element abundances without resorting to a star cluster embedded in its own dark matter halo.

Baumgardt et al. (2009) have ruled out more than $10^{7} M_{\odot}$ of dark matter within $500 \mathrm{pc}$ of the center of NGC 2419. Their upper limit depends on the assumption of isotropic stellar orbits and no mass segregation. In a survey of 10 halo GCs, Lane et al. (2010), extending via their much larger samples earlier work, have found none with $M / L_{V}>5$. These measurements show that the central, most luminous regions of GCs, including NGC 2419, are not dominated by dark matter. However, it is possible that the stellar component of NGC 2419 (and possibly other GCs) formed in a dense dark matter halo but no longer reside at the center of the halo or are significantly more compact than the dark matter distribution.

We ask how such a small, but non-zero range in metallicity could have developed in such a metal-poor system. We require a situation where star formation commenced, but before long, it was quenched. Since prompt initial enrichment is characteristic of essentially all chemical evolution models, this implies that almost all the cluster gas was lost early, thus halting star formation in the accreted satellite dwarf galaxy whose central region eventually became NGC 2419 . How could this have happened given that the $\mathrm{GC}$ is so far out in the halo at the present time? The answer may lie in its orbit, which Casetti-Dinescu et al. (2009) suggest is extremely eccentric, with pericenter at $\sim 11 \mathrm{kpc}$ and apocenter at $\sim 90 \mathrm{kpc}$. Such an orbit would be quite disruptive for the gas, and star formation might cease completely after only one or two orbital periods. Since the period calculated by Casetti-Dinescu et al. (2009) is short, only $1.2 \pm 0.5 \mathrm{Gyr}$, star formation in NGC 2419 could have been terminated quite rapidly.

We are grateful to the many people who have worked to make the Keck Telescope and its instruments a reality and to operate and maintain the Keck Observatory. The authors wish to extend special thanks to those of Hawaiian ancestry on whose sacred mountain we are privileged to be guests. Without their generous hospitality, none of the observations presented herein would have been possible. We thank the anonymous referee for helpful suggestions. J.G.C. thanks NSF grant AST-0908139 for partial support. Work by E.N.K. was supported by NASA through Hubble Fellowship grand HST-HF-01233.01 awarded to ENK by the Space Telescope Science institute, which is operated by the Association of Universities for Research in Astronomy, Inc., for NASA, under contract NAS 5-26555. The analysis pipeline used to reduce the DEIMOS data was developed at UC Berkeley with support from the NSF grant AST-0071048.

\section{REFERENCES}

Baumgardt, H., Côté, P., Rejkuba, M., Mieske, S., Djorgovski, S. G., \& Stetson, P. 2009, MNRAS, 396, 2051

Blecha, A., Meylan, G., North, P., \& Royer, F. 2004, A\&A, 419, 533

Carollo, D., et al. 2007, Nature, 450, 1020

Carollo, D., et al. 2010, ApJ, 712, 692

Carretta, E., et al. 2009, A\&A, 505, 117

Casetti-Dinescu, D. I., et al. 2009, ApJ, 701, L29

Cohen, J. G., Briley, M. M., \& Stetson, P. B. 2005, ApJ, 130, 1177

Cohen, J. G., \& Melendez, J. 2005, AJ, 129, 303

Cohen, J. G., et al. 2008, ApJ, 672, 320

Conroy, C., \& Spergel, D. N. 2010, ApJ, in press

Côté, P., Djorgovski, S. G., Meylan, G., Castro, S., \& McCarthy, J. K. 2002, ApJ, 574,783

Da Costa, G. S., Held, E. V., Saviane, I., \& Gullieuszik, M. 2009, ApJ, 705, 1481

Faber, S. M., et al. 2003, Proc. SPIE, 4841, 1657

Gratton, R., Sneden, C., \& Carretta, E. 2004, ARA\&A, 42, 385

Harris, W. E. 1996, AJ, 112, 1487

Harris, W. E., et al. 1997, AJ, 114, 1030

Ibata, R. A., Gilmore, G. F., \& Irwin, M. J. 1995, MNRAS, 277, 781

Kirby, E. N., Guhathakurta, P., \& Sneden, C. 2008, ApJ, 682, 1217

Kirby, E. N., et al. 2010, ApJS, submitted

Lane, R. R., et al. 2010, MNRAS, 406, 2732

Lee, J. W., Kang, Y. W., Lee, J., \& Lee, Y. W. 2009, Nature, 462, 480

Ma, J., et al. 2007, MNRAS, 376, 1621

Mackey, A. D., \& Gilmore, G. F. 2004, MNRAS, 355, 504

Mackey, A. D., \& van den Bergh, S. 2005, MNRAS, 360, 631

Mackey, D., et al. 2010, ApJ, 717, L11 
Marino, A. F., Milone, A. P., Piotto, G., Villanova, S., Bedin, L. R., Bellini, A., \& Renzini, A. 2009, A\&A, 505, 1099

Milone, A. P., et al. 2008, ApJ, 673, 241

Norris, J. E., Freeman, K. C., \& Mighell, K. J. 1996, ApJ, 462, 241

Olszewski, E. W., Pryor, C., \& Schommer, R. B. 1993, in ASP Conf. Ser. 48 , The Globular Cluster-Galaxies Connection, ed. G. H. Smith \& J. P. Brodie (San Francisco, CA: ASP), 99

Piotti, G. 2009, in IAU Symp. 258, The Ages of Stars, ed. E. E. Mamajek, D. R. Soderblom, \& R. F. G. Wyse (Cambridge: Cambridge Univ. Press)

Racine, R., \& Harris, W. 1975, ApJ, 196, 413

Robin, A. C., Reylé, C., Derriére, S., \& Picard, S. 2003, A\&A, 409, 523

Sandquist, E. L., \& Hess, J. M. 2008, AJ, 36, 2259

Sarajedini, A., \& Layden, A. C. 1995, AJ, 109, 1086

Sarajedini, A., et al. 2007, AJ, 133, 1658

Searle, L., \& Zinn, R. 1978, ApJ, 225, 357
Shetrone, M., Côté, P., \& Sargent, W. L. W. 2001, ApJ, 568, 592

Shortridge, K. 1993, in ASP Conf. Ser. 52, Astronomical Data Analysis Software and Systems II, ed. R. J. Hannisch, R. J. V. Brissenden, \& J. Barnes (San Francisco, CA: ASP), 21

Siegel, M. H., Majewski, S. R., Cudworth, K. M., \& Takamiya, M. 2001, AJ, 121,935

Simon, J. D., \& Geha, M. 2007, ApJ, 670, 313

Sneden, C., Kraft, R. P., Guhathakurta, P., Peterson, R. C., \& Fulbright, J. P. 2004, AJ, 127, 2162

Stetson, P. B. 2005, PASP, 117, 563

Suntzeff, N., Kraft, R. P., \& Kinman, T. D. 1988, AJ, 95, 91

van den Bergh, S., \& Mackey, D. 2004, MNRAS, 354, 713

Vogt, S. E., et al. 1994, Proc. SPIE, 2198, 362

Yi, S., Kim, Y.-C., Demarque, P., \& Alexander, D. R. 2003, ApJS, 143, 499

Zinn, R. 1996, in ASP Conf. Ser. 92, Formation of the Galactic Halo, ed. H. L. Morrison \& A. Sarajedini (San Francisco, CA: ASP), 211 